\title{
SORÇÃO DO INSETICIDA TIAMETOXAM EM LATOSSOLOS SOB EFEITO DE FOSFATO E VINHAÇA
}

\author{
Vitória de Souza de Oliveira*, José Maria de Lima e Renato Fráguas de Carvalho \\ Departamento de Ciência do Solo, Universidade Federal de Lavras, CP 3037, 37200-000 Lavras - MG, Brasil \\ Renê Luís Oliveira Rigitano \\ Departamento de Entomologia, Universidade Federal de Lavras, CP 3037, 37200-000 Lavras - MG, Brasil
}

Recebido em 9/6/08; aceito em 27/1/09; publicado na web em 3/7/09

\begin{abstract}
SORPTION OF THE INSECTICIDE THIAMETHOXAM IN LATSOLS UNDER THE EFFECT OF BOTH PHOSPHATE AND VINASSE. Little information is available on the behavior of thiamethoxam in soils, whereas many studies show the effect of phosphate and vinasse in soils in Brazil. This study evaluated the sorption, desorption and retention of thiamethoxam in vinasse- and phosphate-amended samples of a dystrophic Red-Yellow Latosol (LVAd) and a distroferric Red Latosol (LVdf). The LVAd presented higher sorption of thiamethoxam. Phosphate did not affect the sorption or retention and vinasse increased the interaction of the compound with the soil particles, reducing desorption to the soil solution.
\end{abstract}

Keywords: constant of Freundlich; retention; pesticide.

\section{INTRODUÇÃO}

O tiametoxam, um inseticida pertencente à classe dos neonicotinoides, foi recentemente registrado no Brasil para o controle de pragas em várias culturas, especialmente no cafeeiro, onde é aplicado via solo para o controle do bicho-mineiro. O produto é comercializado como Actara ${ }^{\circledR}$, empregado para aplicação no solo e pulverização foliar, e como Cruiser ${ }^{\circledR}$, para tratamento de sementes. Trata-se de um composto altamente tóxico a insetos e pouco tóxico a mamíferos, apresentando DL 50 para ratos (dose letal a $50 \%$ dos indivíduos tratados) em torno de $1500 \mathrm{mg} \mathrm{kg}^{-1}$ de peso corporal. ${ }^{1}$ Entretanto, quando em contato constante, mesmo em pequenas doses, esse inseticida tem grande potencial carcinogênico, sendo seu limite aceitável para impacto na água do solo de $0,94 \mu \mathrm{g} \mathrm{\textrm {L } ^ { - 1 }} .^{2}$

Devido a suas propriedades físico-químicas, o tiametoxam apresenta baixa sorção aos colóides do solo, estando mais relacionado com o teor de matéria orgânica do solo, apresentando dessa forma alto potencial de lixiviação em condições de campo. ${ }^{2-5}$ Pouco ainda se sabe sobre o comportamento do tiametoxam em solos tropicais, principalmente com relação a algumas práticas de manejo do solo, como a fosfatagem e a aplicação de vinhaça ao solo. Tanto o fosfato quanto a vinhaça exercem efeitos sobre alguns atributos físico-químicos do solo e, consequentemente, influenciam a dinâmica de moléculas de pesticidas em solos. A vinhaça é um resíduo do processo de destilação do álcool e consiste numa fonte de carbono solúvel, sendo o glicerol seu principal constituinte. ${ }^{6}$ Sua adição ao solo modifica, temporariamente, alguns atributos como $\mathrm{pH}$, quantidade de $\mathrm{C}$ orgânico e atividade e biomassa microbiana. ${ }^{6-9} \mathrm{~A}$ fosfatagem altera a dispersão de partículas, o balanço de cargas, ${ }^{10}$ além do conteúdo e a dissolução da matéria orgânica no solo e a liberação de matéria orgânica adsorvida à superfície de partículas minerais do solo. ${ }^{11,12}$

Neste sentido o presente trabalho objetivou estudar o efeito da vinhaça e fosfatagem nos processos de adsorção, dessorção e retenção do inseticida tiametoxam em Latossolo Vermelho Amarelo distrófico e Latossolo Vermelho distroférrico.

\section{PARTE EXPERIMENTAL}

As amostras de solo foram coletadas na camada de $0-10 \mathrm{~cm}$ de um Latossolo Vermelho Amarelo distrófico-LVAd e Latossolo Vermelho

*e-mail: vitoriasoliveira@ig.com.br
distroférrico-LVdf, do município de Lavras, MG, e caracterizadas quimicamente, de acordo com Embrapa (1997) e pela granulometria das partículas, pelo método da pipeta (Tabela 1). ${ }^{13,14}$

A molécula do tiametoxam, nome químico (IUPAC) (EZ)3-(2-clorotiazol-1,3-tiazol-5-ilmetil)-5-metil-1,3,5-oxadiazinan-4-ilideno(nitro) amina, é ilustrada na Figura 1. O composto possui solubilidade em água a $25^{\circ} \mathrm{C}$ equivalente a $4,1 \mathrm{~g} \mathrm{~L}^{-1}$ e Log Kow de $-0,13 .{ }^{15,16}$<smiles>CN1COCN(Cc2cnc(Cl)s2)/C1=N\[O+]([O-])[O-]</smiles>

Figura 1. Estrutura química do tiametoxam

A solução estoque do composto utilizada nos ensaios foi obtida a partir da purificação do produto comercial Actara ${ }^{\circledR}$, que contém $25 \%$ do composto. Diluíram-se $100 \mathrm{mg}$ do Actara $^{\circledR} \mathrm{em}$ acetona, filtrada em algodão para um balão volumétrico de $100 \mathrm{~mL}$. A acetona foi evaporada por meio de rotavapor. Adicionou-se, então, aproximadamente, $1 \mathrm{~mL}$ de acetona no balão e, em seguida, essa solução foi transferida para duas placas de TLC (sílica gel $60 \mathrm{GF}_{254}$ Merck), com auxílio de uma pipeta Pasteur. A fase móvel utilizada foi diclorometano:acetona, na proporção 100:150. A faixa contendo o tiametoxam foi identificada com luz UV e eluída em acetona. A concentração de tiametoxam foi determinada em cromatografia líquida de alta eficiência (CLAE), a partir do padrão analítico com $99 \%$ de pureza.

\section{Cinética de sorção do tiametoxam}

A cinética de sorção do tiametoxam foi avaliada segundo a metodologia descrita por Felsot e Dahm, ${ }^{17}$ transferindo-se $3 \mathrm{~g}$ de material de solo para tubos de vidro de $20 \mathrm{~mL}$ com tampa rosqueável, adicionando-se, em seguida, $10 \mathrm{~mL}$ de solução aquosa de $\mathrm{CaCl}_{2}$ $0,01 \mathrm{M}$, contendo tiametoxam na concentração de $2 \mathrm{mg} \mathrm{L}^{-1}$.

Os tubos foram submetidos à agitação horizontal constante com 120 movimentos por min durante 0,$25 ; 0,5 ; 1 ; 1,5 ; 2 ; 3 ; 4 ; 6 ; 12$ e 24 h. Ao final de cada tempo de agitação a suspensão foi centrifugada 
Tabela 1. Caracterização química e granulométrica das amostras de Latossolo Vermelho Amarelo distrófico (LVAd) e Latossolo Vermelho distroférrico (LVdf), da região Sul de Minas Gerais

\begin{tabular}{|c|c|c|c|c|c|c|c|c|c|c|c|c|c|c|c|}
\hline Solo & $\mathrm{pH}^{1}$ & $\mathrm{P}^{2}$ & $\mathrm{~K}^{+}$ & $\mathrm{Ca}^{2+}$ & $\mathrm{Mg}^{2+}$ & $\mathrm{Al}^{2+}$ & $\mathrm{SB}^{3}$ & $t^{4}$ & $\mathrm{~T}^{5}$ & $\mathrm{MO}^{8}$ & Argila & Areia & $\mathrm{Cmax}^{9}$ & $\mathrm{CC}^{10}$ & $\mathrm{VP}^{11}$ \\
\hline & $\mathrm{H}_{2} \mathrm{O}$ & \multicolumn{2}{|c|}{$\mathrm{mg} \mathrm{dm}^{-3}$} & - & & $--\mathrm{cm}$ & $\mathrm{dm}^{-3}$ & --- & ---- & ----- & $-\mathrm{g} \mathrm{kg}^{-1}$ & ---- & $\mathrm{mg} \mathrm{kg}^{-1}$ & \multicolumn{2}{|c|}{---- \% ---- } \\
\hline LVAd & 5,8 & 3,5 & 65 & 2,8 & 0,4 & 0,1 & 3,4 & 3,5 & 8,2 & 30 & 481,1 & 362,4 & 714,3 & 34,3 & 58,5 \\
\hline LVdf & 5,7 & 4,1 & 84,3 & 2,0 & 0,6 & 0,1 & 2,7 & 2,8 & 6,6 & 26 & 594,3 & 184,3 & 625,0 & 40,3 & 59,6 \\
\hline
\end{tabular}

${ }^{1} \mathrm{pH}$ em água: relação $(1: 2,5) ;{ }^{2} \mathrm{P}$ extraído por Mehlich-1; ${ }^{3} \mathrm{SB}$, soma de bases trocáveis; ${ }^{4} \mathrm{t}$, capacidade de troca catiônica efetiva; ${ }^{5} \mathrm{~T}$, capacidade de troca de cátions a pH 7,0; ${ }^{6} \mathrm{~V}$, índice de saturação por bases; ${ }^{7} \mathrm{~m}$, índice de saturação por alumínio; ${ }^{8} \mathrm{MO}$, matéria orgânica; ${ }^{9} \mathrm{Cmax}$, capacidade máxima de adsorção de fósforo; ${ }^{10} \mathrm{CC}$, capacidade de campo $(0,10 \mathrm{~atm}) ;{ }^{11} \mathrm{VP}$, volume total de poros

a $710 \mathrm{~g}$ por $30 \mathrm{~min}$, retirando-se uma alíquota de $2 \mathrm{~mL}$ da fase aquosa, que foi filtrada em membrana de 0,45 $\mu \mathrm{m}$ de PTFE, com auxílio de uma seringa de vidro, e armazenada em geladeira para posterior análise por CLAE. A concentração do tiametoxam no solo foi calculada pela diferença entre a concentração inicial e a concentração do composto encontrada na fase aquosa após o respectivo tempo de agitação, multiplicada pelo volume de solução e dividida pela massa de solo.

\section{Tratamentos das amostras com fosfato e vinhaça}

As amostras foram incubadas com calcário (PRNT 100\%), durante 1 mês, suficiente para elevar o pH das amostras de solo a 6,0. Após esse período, um terço das amostras recebeu fosfato suficiente para se manter $0,2 \mathrm{mg} \mathrm{L}^{-1}$ de $\mathrm{P}$ na solução do solo, um terço recebeu fosfato suficiente para se atingir $50 \%$ da capacidade máxima de adsorção de fósforo (Cmax) dos solos e o restante não recebeu fosfato; foi empregado fosfato de potássio monobásico $\left(\mathrm{KH}_{2} \mathrm{PO}_{4}\right)$. As amostras permaneceram incubadas por mais 1 mês, com umidade mantida a $60 \%$ da capacidade de campo. Em seguida, foram estabelecidas três condições de vinhaça para cada condição de fosfato descrita anteriormente: zero, 50 e $70 \%$ do volume total de poros (VP) das amostras, calculado a partir do volume, densidade de partículas e massa do solo no vaso no qual as amostras foram incubadas. As amostras permaneceram incubadas com vinhaça durante 15 dias, com umidade do solo mantida em torno de $70 \%$ do volume total de poros. As características químicas da vinhaça encontram-se descritas na Tabela 2.

\section{Isotermas de sorção e dessorção do tiametoxam}

Para determinação das isotermas de sorção, tubos de vidro de 20 $\mathrm{mL}$ contendo $3 \mathrm{~g}$ de amostra de cada tratamento receberam $10 \mathrm{~mL}$ da solução de $\mathrm{CaCl}_{2}$,, $01 \mathrm{M}$ contendo tiametoxam nas concentrações 0,1 ; 0,5; 1,0; 5,0 e 10,0 $\mathrm{mg} \mathrm{L}^{-1}$, com quatro repetições por concentração. Os tubos foram agitados durante $4 \mathrm{~h}$, medindo-se a sorção do tiametoxam no solo em seguida, como descrito para cinética de sorção.

Para o estudo da dessorção, logo após a centrifugação e retirada da solução sobrenadante, os tubos foram pesados, para se determinar a quantidade de solução oclusa e, em seguida, cada tubo recebeu 10 $\mathrm{mL}$ da solução de $\mathrm{CaCl}_{2} 0,01 \mathrm{M}$, foram agitados durante $4 \mathrm{~h}$ e centrifugados a $710 \mathrm{~g}$, por $30 \mathrm{~min}$, retirando-se $2 \mathrm{~mL}$ da solução aquosa para posterior análise por CLAE.
O modelo de Freundlich $\left(S=K f C e^{l / n}\right)$ foi empregado para obtenção dos parâmetros de sorção e dessorção (Kf e grau de linearidade $1 / n)$. Nessa equação, $S$ representa a concentração de equilíbrio do pesticida na fase sólida do solo $\left(\mathrm{mg} \mathrm{kg}^{-1}\right), \mathrm{Ce}$ a concentração de equilíbrio do pesticida na solução do solo $\left(\mathrm{mg} \mathrm{L}^{-1}\right), K f$ a constante de equilíbrio de Freundlich $\left[\left(\mathrm{mg} \mathrm{kg}^{-1}\right)\left(\mathrm{L} \mathrm{mg}^{-1}\right)^{1 / \mathrm{n}}\right]$ e $1 / n$ o grau de linearidade da isoterma. ${ }^{18}$

\section{Condições operacionais do cromatógrafo}

Para determinação quantitativa do tiametoxam, foi utilizado um sistema de cromatografia líquida de alta eficiência (CLAE), HP série 1100, operando com detector de arranjo de diodo (DAD) no comprimento de onda de $255 \mathrm{~nm}$. A coluna utilizada foi uma Waters Spherisorb ODS-2, $5 \mu \mathrm{m}, 150$ x 3,2 mm. A fase móvel utilizada foi acetonitrila/água milli-Q, na proporção 20:80, com fluxo de $0,3 \mathrm{~mL}$ min $^{-1}$ e volume de injeção de $20 \mu \mathrm{L}$. Nessas condições, o tempo de retenção do tiametoxam ficou em torno de 8,20 min. A quantificação da concentração do tiametoxam foi realizada por comparação da área dos picos das amostras com a curva padrão de calibração, utilizandose na sua construção o padrão analítico do tiametoxam. O padrão analítico utilizado foi obtido junto à Syngenta Proteção de Cultivos Ltda., tendo especificação de pureza acima de $99 \%$.

\section{Análise estatística}

Devido à falta de normalidade, os dados de $\mathrm{Kf}$ das isotermas de Freundlich, encontrados para cada tratamento, foram transformados em $\log (\mathrm{x})$. Após a transformação, realizou-se análise de variância da interação $2 \times 3 \times 3$ (dois solos $x$ três doses de vinhaça $x$ três doses de fósforo) e as médias foram comparadas pelo teste de Tukey a $5 \%$ de significância.

\section{RESULTADOS E DISCUSSÃO}

\section{Cinética de sorção do tiametoxam}

Os resultados do estudo de cinética do tiametoxam estão apresentados na Figura 2. Os solos estudados apresentaram comportamentos semelhantes entre si, com relação ao tempo requerido para atingir o equilíbrio. Após 1 h sob agitação constante, a concentração

Tabela 2. Características químicas da vinhaça

\begin{tabular}{|c|c|c|c|c|c|c|c|c|c|}
\hline $\mathrm{pH}$ & $\mathrm{C}^{1}$ & $\mathrm{P}^{2}$ & $\mathrm{~K}^{2}$ & $\mathrm{Ca}^{2}$ & $\mathrm{Mg}^{2}$ & $\mathrm{Cu}^{2}$ & $\mathrm{Mn}^{2}$ & $\mathrm{Fe}^{2}$ & $\mathrm{Zn}^{2}$ \\
\hline & ------. & - & $-\mathrm{g} \mathrm{L}^{-1}$ & ------ & ------- & -----. & ------- & ------ & ----- \\
\hline 3,5 & 2,86 & 0,13 & 0,22 & 0,14 & 0,03 & 1,91 & 0,63 & 9,95 & 1,33 \\
\hline
\end{tabular}

${ }^{1}$ Determinado de acordo com o método descrito em Yeomans \& Bremner (1988), adaptado; ${ }^{2}$ a caracterização química da vinhaça foi feita após digestão nitroperclórica, sendo $\mathrm{Fe}, \mathrm{Zn}, \mathrm{Cu}, \mathrm{Mg}, \mathrm{Mn}$ e Ca medidos por absorção atômica, $\mathrm{P}$ por espectroscopia de luz e K por espectrofotometria de chama 


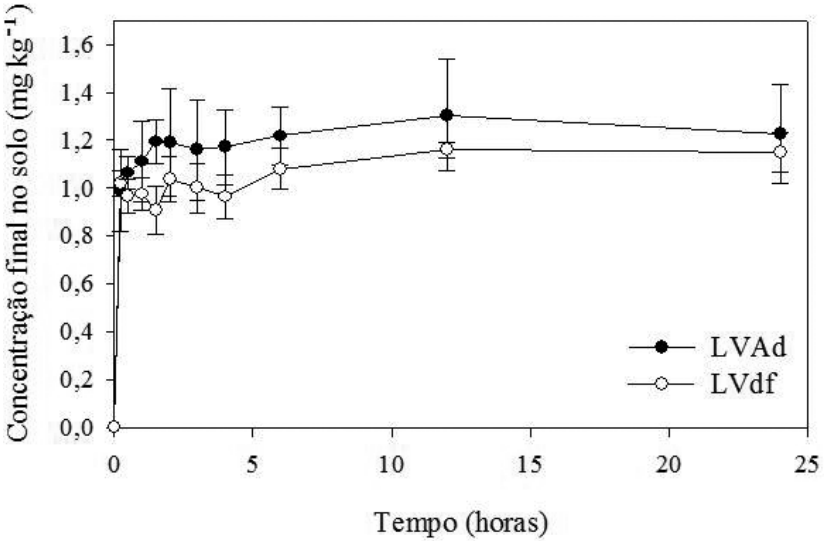

Figura 2. Efeito do tempo de agitação sobre a sorção do tiametoxam em amostras de um Latossolo Vermelho Amarelo distrófico (LVAd) e Latossolo Vermelho distroférrico (LVdf) de tiametoxam no conjunto solo-solução atingiu o equilíbrio. Resultados semelhantes foram encontrados por Urzedo, ${ }^{20} \mathrm{em}$ amostras de um Latossolo Vermelho Amarelo distroférrico. Entretanto, por motivo de segurança, no tocante a se estabelecer o equilíbrio entre solo e solução, o tempo utilizado nos ensaios de sorção e de dessorção foi de $4 \mathrm{~h}$.

\section{Efeito da vinhaça e fosfato na sorção e dessorção do tiametoxam}

As isotermas de sorção e dessorção, ajustadas ao modelo de Freundlich, obtidas para o tiametoxam, em amostras de LVAd e LVdf sob efeito da vinhaça e fosfato, tiveram coeficientes de determinação $\left(r^{2}\right)$ variando entre 0,79 e 0,99 . Os valores das constantes de Freundlich (Kf) e dos coeficientes de linearidade (1/n) encontram-se na Tabela 3. A sorção do tiametoxam no LVAd foi, significativamente, superior àquela observada no LVdf para todos os tratamentos. O LVAd

Tabela 3. Valores da constante de Freundlich (Kf), coeficiente de linearidade $(1 / \mathrm{n})$ e coeficiente de determinação $\left(\mathrm{r}^{2}\right)$ para a sorção e dessorção do tiametoxam em amostras de Latossolo Vermelho Amarelo distrófico e Latossolo Vermelho distroférrico sob o efeito da vinhaça e fosfatagem

\begin{tabular}{lllccc}
\hline & Isoterma de sorção & & \multicolumn{2}{c}{ Isoterma de dessorção } \\
Kf* & $1 / n$ & $\mathrm{r}^{2}$ & $\mathrm{Kf} *$ & $1 / \mathrm{n}$ & $\mathrm{r}^{2}$ \\
\hline
\end{tabular}

\section{LVAd}

Vinhaça $=0$

Vinhaça 50\% VP

Vinhaça $70 \%$ VP

Vinhaça $=0$

Vinhaça 50\% VP

Vinhaça 70\% VP

Vinhaça $=0$

Vinhaça 50\% VP

Vinhaça $70 \%$ VP

\section{LVdf}

Vinhaça $=0$

Vinhaça 50\% VP

Vinhaça $70 \%$ VP

0,69 a

1,07 a

0,96 a

$1,13 \mathrm{a}$

$1,07 \mathrm{ab}$

$0,96 \mathrm{~b}$

1,16 a

$1,08 \mathrm{ab}$

$0,94 \mathrm{~b}$

$1,20 \mathrm{a}$

Dose de $\mathrm{P}=0$

$0,99 \pm 0,10 \quad 0,97$

0,97
0,98

$11,61 \mathrm{~b}$

$1,30 \pm 0,12$

0,92

$1,03 \pm 0,01$

0,98

24,42 a

$1,08 \pm 0,06$

0,93

$0,86 \pm 0,05$

0,95

23,83 a

$1,06 \pm 0,10$

0,85

Dose de $\mathrm{P}=0,2 \mathrm{mg} \mathrm{L}^{-1}$

$\begin{array}{lllll}0,99 \pm 0,02 & 0,98 & 11,09 \mathrm{~b} & 1,27 \pm 0,09 & 0,91\end{array}$

$1,01 \pm 0,02$

0,98

19,38 a

$0,97 \pm 0,17$

0,87

$0,85 \pm 0,02$

0,96

23,38 a

$1,12 \pm 0,07$

0,92

Dose de $\mathrm{P}=50 \%$ CMAX

$\begin{array}{lllll}0,99 \pm 0,01 & 0,98 & 13,34 \mathrm{a} & 1,35 \pm 0,01 & 0,94 \\ 1,03 \pm 0,03 & 0,98 & 20,74 \mathrm{a} & 1,01 \pm 0,07 & 0,91 \\ 0,87 \pm 0,03 & 0,96 & 18,50 \mathrm{a} & 1,00 \pm 0,06 & 0,77\end{array}$

Dose de $\mathrm{P}=0$

Vinhaça $=0$

$0,71 \mathrm{a}$
$0,75 \mathrm{a}$
$0,77 \mathrm{a}$

$0,76 \pm 0,01$

0,97

$2,90 \mathrm{~b}$

$0,72 \pm 0,14$

0,97

$0,75 \pm 0,03$

0,99

$2,73 \mathrm{~b}$

$0,54 \pm 0,10$

0,97

$0,86 \pm 0,02$

0,99

5,88 a

$0,87 \pm 0,07$

0,95

Dose de $\mathrm{P}=0,2 \mathrm{mg} \mathrm{L}^{-1}$

Vinhaça 50\% VP

$0,79 \pm 0,04$

0,99

$2,72 \mathrm{~b}$

$0,72 \pm 0,16$

0,94

Vinhaça $70 \%$ VP

$0,77 \mathrm{a}$

$0,70 \pm 0,14$

0,96

$3,11 \mathrm{~b}$

$0,60 \pm 0,05$

0,96

$0,85 \pm 0,01$

5,42 a

$0,84 \pm 0,07$

0,95

Dose de $\mathrm{P}=50 \%$ CMAX

Vinhaça $=0$

0,75 a

$0,75 \pm 0,02$

0,99

$2,41 \mathrm{~b}$

$0,61 \pm 0,09$

0,98

Vinhaça $50 \%$ VP

0,68 a

$0,77 \pm 0,02$

0,99

$3,38 \mathrm{ab}$

$0,61 \pm 0,11$

0,93

0,79 a

$0,83 \pm 0,03$

0,99

5,51 a

$0,86 \pm 0,06$

0,95

* Kf (mg $\left.{ }^{1-1 / n} \mathrm{~L}^{1 / n} \mathrm{~kg}^{-1}\right)$. Interação tripla significativa a 1\%, com coeficiente de variação de 7,24\% para os valores de Kf da sorção e $23,66 \%$ para os valores de $\mathrm{Kf}$ da dessorção. Fixando-se as doses de vinhaça e fósforo observou-se diferença significativa (5\%) entre os dois solos pelo teste de Tukey. Fixando-se as doses de vinhaça e o solo não foi observada diferença significativa (5\%) entre as três doses de fósforo pelo teste de Tukey. Médias com letras diferentes na coluna, para mesma dose de fósforo e solo, diferem significativamente (5\%) entre si pelo teste de Tukey. 
apresentou maiores valores de Kf, significando menor potencial de lixiviação do produto nesse solo em condições de equilíbrio.

A presença de fosfato não influenciou os processos de sorção e dessorção do tiametoxam nos solos estudados. Quanto à vinhaça, a maior dose, $70 \% \mathrm{VP}$, apenas causou acréscimo significativo na sorção do tiametoxam nas amostras de LVAd com aplicação de P. Tendências de valores de Kf ligeiramente superiores foram observadas nas demais amostras tratadas com essa dose de vinhaça, porém as diferenças não foram significativas. A pouca interferência da adição da vinhaça na sorção do tiametoxam é atribuída ao fato de a matéria orgânica desse resíduo ser constituída, principalmente, de glicerol, que é prontamente disponível aos micro-organismos quimio-organotróficos do solo. Alguns autores afirmam que, após 4 dias da adição da vinhaça no solo, grande parte da matéria orgânica do resíduo é mineralizada. ${ }^{8,21}$

A sorção do tiametoxam foi parcialmente reversível para os dois solos estudados. Porém, os valores de Kf da dessorção foram maiores que os valores de Kf da sorção, principalmente por se tratar de um único ciclo de extração para se determinar a dessorção. Essa diferença indica que a remoção do produto sorvido ao solo é baixa. Esse fenômeno, conhecido como histerese, indica que diferentes forças de interação estão envolvidas nos processos de sorção e dessorção. A histerese tem sido frequentemente observada na sorção de pesticidas em solos, ${ }^{17,22-24}$ incluindo o tiametoxam..${ }^{4,5,20}$ A presença de vinhaça contribuiu para aumentar as forças de interação na dessorção, propiciando maior retenção do inseticida no solo, principalmente nas amostras tratadas com a dose correspondente a $70 \% \mathrm{VP}$, para os dois solos estudados; esse aumento da retenção fica evidenciado com os maiores valores de $\mathrm{Kf}$ encontrados nas amostras tratadas com a maior dose de vinhaça. Os maiores valores de Kf encontrados da dessorção indicam que, embora a vinhaça não tenha propiciado aumento significativo na sorção em todas as amostras, quando está presente nos solos, principalmente no LVdf, dificulta a dessorção do produto para a solução.

\section{CONCLUSÕES}

O tiametoxam revelou-se um inseticida com baixa sorção e parcialmente reversível em amostras de Latossolo Vermelho Amarelo distrófico (LVAd) e Latossolo Vermelho distrófico (LVdf).

A fosfatagem não afetou os processos de sorção e dessorção do tiametoxam em amostras do LVAd e LVdf.

A vinhaça exerceu pouca influência no processo de sorção do tiametoxam nas amostras de LVAd e LVdf. Entretanto, sua adição aumentou a retenção do composto nos solos, após a dessorção.

\section{MATERIAL SUPLEMENTAR}

A visualização dos efeitos da vinhaça e do fosfato na sorção e dessorção do tiametoxam em amostras de LVAd e LVdf fica mais clara quando observa-se suas isotermas, ajustadas ao modelo de Freundlich, Figuras $1 \mathrm{~S}$ e $2 \mathrm{~S}$. Este material encontra-se disponível em http://quimicanova. sbq.org.br, na forma de arquivo PDF, com acesso livre.

\section{AGRADECIMENTOS}

À Fapemig e ao CNPq pelo apoio financeiro.

\section{REFERÊNCIAS}

1. Antunes-Kenyon, S. E.; Kennedy, G.; Tiametoxam: a new active ingredient review, Massachusetts Pesticide Bureau: Boston, 2001.

2. http://www.dec.state.ny.us/website/dshm/pesticid/pesticid.htm, acessada em Outubro 2007.

3. Banerjee, K.; Patil, S. H.; Dasgupta, S.; Oulkar, D. P.; Adsule, P. G.; J. Environ. Sci. Health, Part B 2008, 43, 151.

4. Castro, N. R. A.; Tese de Doutorado, Universidade Federal de Lavras, Brasil, 2005.

5. Urzedo, A. P. F. M.; Rigitano, R. L. O.; Lima, J. M.; Castro, N. R. A.; Pesticidas: R. Ecotoxicol. Meio Ambiente 2006, 16, 71.

6. Rodella, A. A.; Zambello Junior E.; Orlando Filho, J.; Resumos do Congress of the International Society of Sugar Cane Technologists, Havana, Cuba, 1983.

7. Camargo, O. A.; Valadares, J. M. A.; Berton, R. S.; Teófilo Sobrinho, J.; Menk, J. R. F. A.; Alteração de características químicas de um latossolo vermelho-escuro distrófico pela aplicação de vinhaça, Instituto Agronômico: Campinas, 1987.

8. Prata, F.; Lavorenti, A.; Regitano, J. B.; Tornisielo, V. L.; Rev. Bras. Ci. Solo 2000, 24, 217.

9. Silva, M. A. S.; Griebeler, N. P.; Borges, L. C.; Rev. Bras. Eng. Agr. Ambiental 2007, 11, 108.

10. Lima, J. M.; Anderson, S. J.; Curi, N.; Soil Sci. Soc. Am. J. 2000, 64, 892.

11. Myers, R.; Thien, S. J.; Soil Sci. Soc. Am. J. 1991, 55, 866.

12. Afif, E.; Barrón, V.; Torrent, J.; Soil Sci. 1995, 159, 207.

13. Empresa Brasileira de Pesquisa Agropecuária; Manual de métodos de análises de solo, $2^{\mathrm{a}}$ ed., CNPS: Rio de Janeiro, 1997.

14. Day, P. R. Em Methods of soil analysis; Black, C. A., ed.; American Society of Agronomy: Madison, 1965, p. 545-566.

15. Maienfisch, P.; Angst, M.; Bradl, F.; Fischer, W.; Hofer, D.; Kayser, H.; Kobel, W.; Rindlisbacher, A.; Senn, R.; Steinemann, A.; Widmer, H.; Pest Manage. Sci. 2001, 57, 906.

16. Novartis.; Tiametoxam 250WG, São Paulo, 1998.

17. Felsot, A.; Dahm, P. A. J.; J. Agric. Food Chem. 1979, 27, 557.

18. Green, R. E.; Karickhoff, S. W. Em Pesticides in the soil environment: processes, impacts, and modeling; Cheng, H. H., ed.; Soil Science Society of America: Madison, 1990, p. 79-101.

19. Ferreira, D. F.; Resumos da Reunião Anual da Região Brasileira da Sociedade Internacional de Biometria, São Carlos, Brasil, 2000.

20. Urzedo, A. P. F. M.; Dissertação de Mestrado, Universidade Federal de Lavras, Brasil, 2004.

21. Prata, F.; Lavonreti, A.; Regitano, J. B.; Tornisielo, V. L.; Pesq. Agropec. Brasileira 2001, 36, 975.

22. Barison, R. R. M.; Lavonreti, A.; Regitano, J. B.; Tornisielo, V. L.; Rev. Bras. Ci. Solo 2005, 29, 695.

23. Ding, G. W.; Novak, J. M.; Herbert, S.; Baoshan, X.; Chemosphere 2002, $48,897$.

24. Gao, J. P.; Maguhn, J.; Spitzauer, P.; Ketrup, A.; Water Res. 1998, 32, 1622. 


\section{SORÇÃO DO INSETICIDA TIAMETOXAM EM LATOSSOLOS SOB EFEITO DE FOSFATO E VINHAÇA}

Vitória de Souza de Oliveira*, José Maria de Lima e Renato Fráguas de Carvalho

Departamento de Ciência do Solo, Universidade Federal de Lavras, CP 3037, 37200-000 Lavras - MG, Brasil

Renê Luís Oliveira Rigitano

Departamento de Entomologia, Universidade Federal de Lavras, CP 3037, 37200-000 Lavras - MG, Brasil
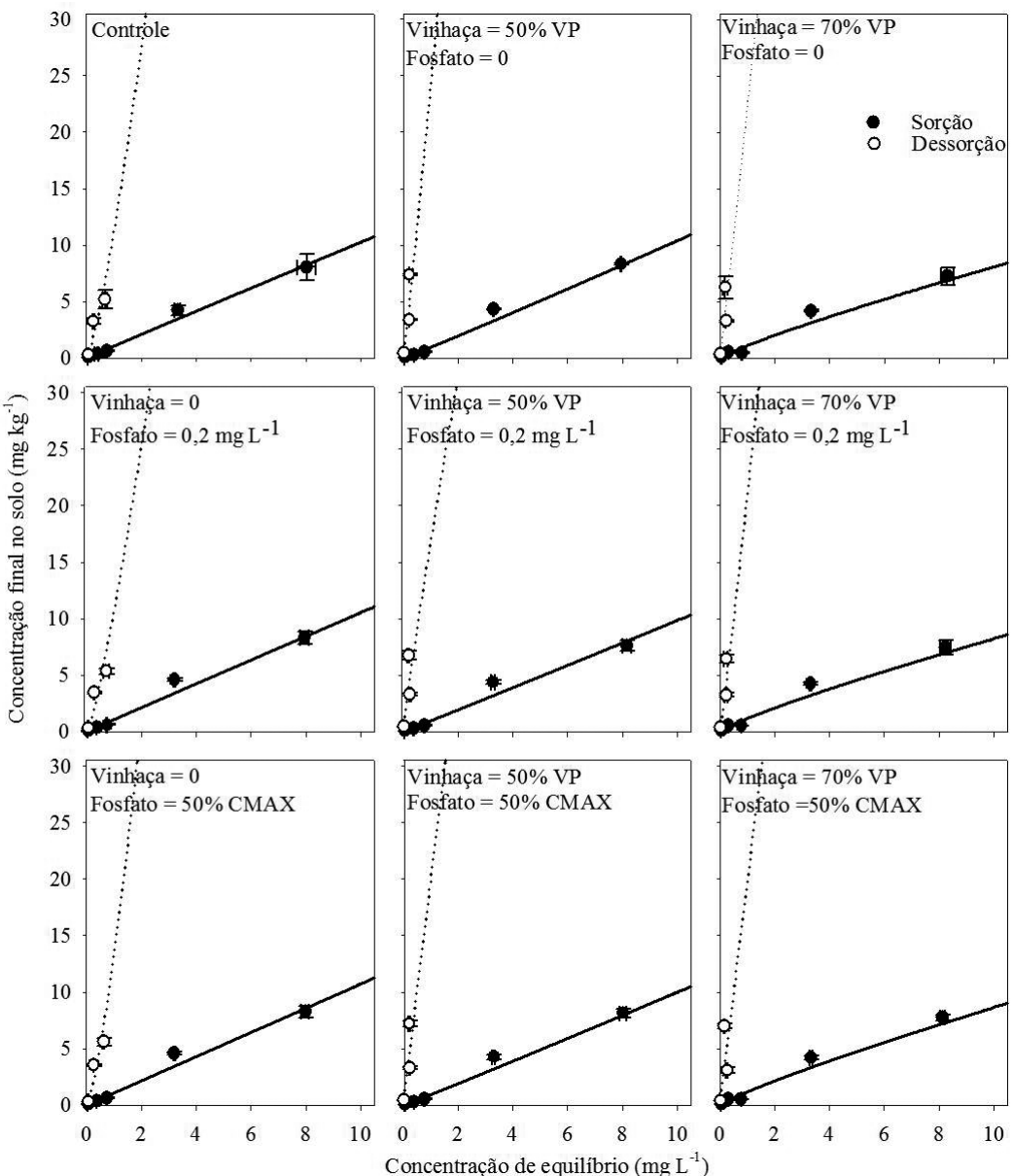

Figura 1S. Isotermas de sorção e dessorção de tiametoxam em amostras de Latossolo Vermelho Amarelo distrófico sob efeito de vinhaça e fosfato 

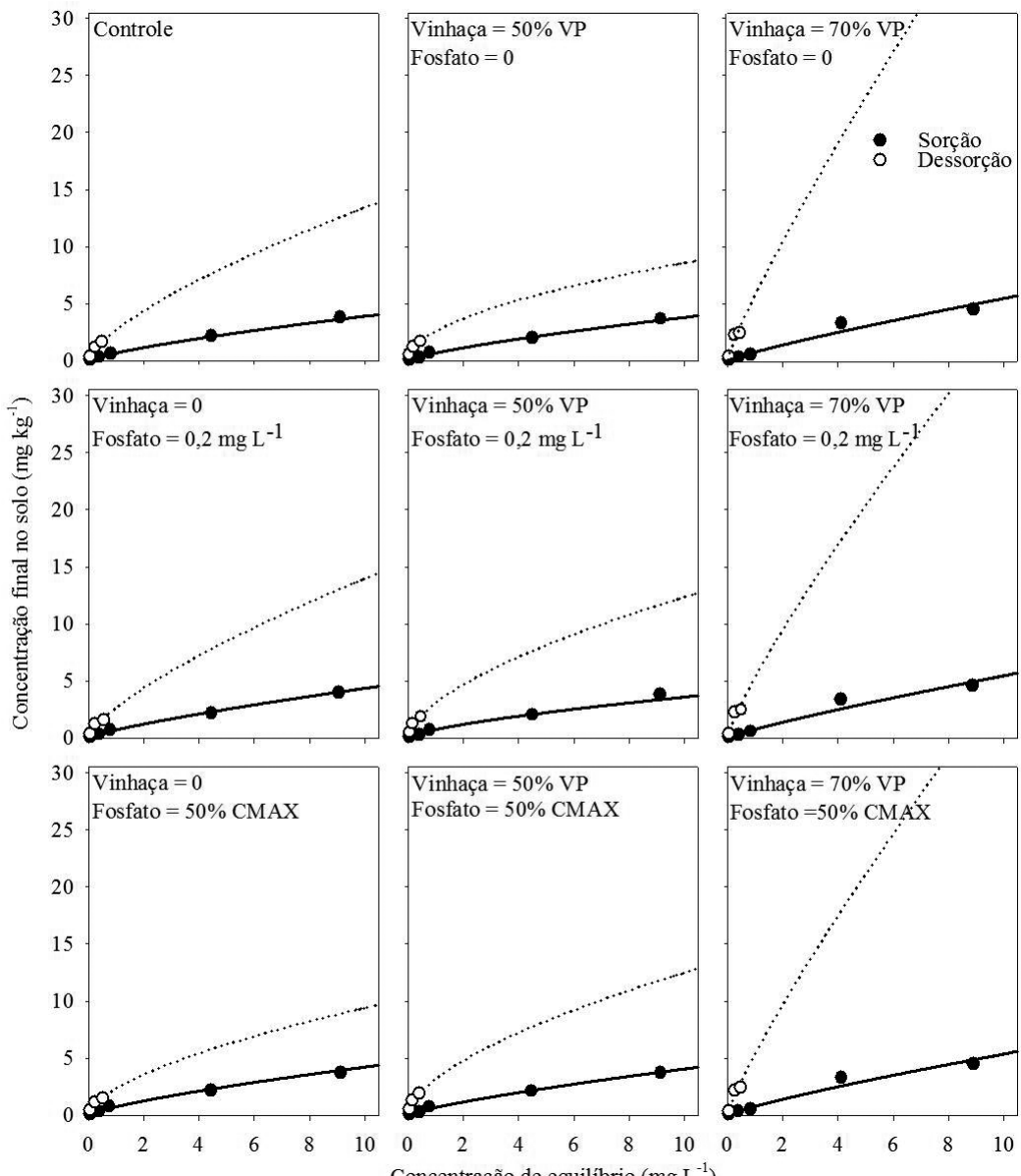

Concentração de equilíbrio $\left(\mathrm{mg} \mathrm{L}^{-1}\right)$

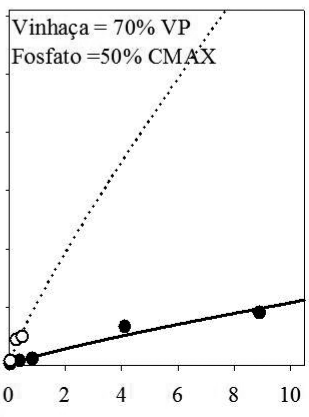

Figura 2S. Isotermas de sorção e dessorção de tiametoxam em amostras de Latossolo Vermelho distroférrico sob efeito de vinhaça e fosfato 